González-Sodis, J.L., Leiva-Olivencia, J.J. \& Matas-Terrón, A. (2021). Percepción de la violencia verbal en las aulas: La mediación en escena. Revista Electrónica Interuniversitaria de Formación del Profesorado, 24(3), 221-236.

\title{
Percepción de la violencia verbal en las aulas: La mediación en escena
}

José Luis González-Sodis, Juan José Leiva-Olivencia, Antonio Matas-Terrón

Universidad de Málaga

\section{Resumen}

En los últimos años estamos contemplando como en prensa los conflictos escolares están a la orden del día. Las administraciones educativas en general y todos los centros educativos de nuestras Comunidades Autónomas están muy preocupados por frenar el avance conflictivo que en muchas escuelas surgen. Con objeto de mitigar estas situaciones se implementa programas que favorecen la convivencia escolar.

El objetivo del presente trabajo es examinar cómo perciben los conflictos escolares una muestra de estudiantes de Secundaria Obligatoria (ESO) de la provincia de Málaga. Se ha realizado un estudio basado en la percepción de conflictos desde la perspectiva de los estudiantes a partir de un Cuestionario de Violencia Escolar (CUVE). La muestra está formada por 1069 estudiantes de centros educativos de la provincia de Málaga. Los resultados obtenidos, indican la existencia de un nivel significativo de la conflictividad. Concluimos que los conflictos analizados entre estudiantes y docentes pueden ser abordados a partir de la mediación escolar. La mediación escolar es una estrategia con principios promotores éticos, voluntariedad, neutralidad de las personas mediadoras y confidencialidad, que repercuten en el desarrollo psicosocial de los estudiantes.

\section{Palabras clave}

Convivencia; conflicto; violencia verbal; mediación. 


\section{Perception of verbal violence in the classroom: Mediation on stage}

\section{Abstract}

In recent years, we have seen how school conflicts have become the order of the day in the press. The educational administrations in general and all the educational centres of our Autonomous Communities are very concerned about curbing the conflictive progress that arises in many schools. In order to mitigate these situations, programmes that favour school coexistence are implemented.

The aim of this paper is to examine how school conflicts are perceived by sample of students in Compulsory Secondary Education (ESO) in the province of Malaga. A study has been carried out based on the perception of conflicts from the students' perspective using a School Violence Questionnaire (CUVE). The sample consisted of 1069 students from schools in the province of Malaga. The results obtained indicate the existence of a significant level of conflict. We conclude that the conflicts analysed between students and teachers can be addressed through school mediation. School mediation is a strategy with promoting ethical principles, voluntariness, neutrality of the mediators and confidentiality, which have an impact on the psychosocial development of students.

\section{Keywords}

Coexistence; conflict; verbal violence; mediation.

\section{Introducción}

Desde hace algún tiempo la preocupación por la convivencia escolar está siendo cada vez más relevante y las administraciones educativas proyectan desde sus competencias programas que persiguen la mejora del clima escolar, fundamentalmente basados en estrategias como la mediación escolar entre iguales (Torrego, 2018; Boqué, 2018; Moreta, 2018; Sánchez et al., 2019; Iriarte et al., 2020).

La concepción de convivencia escolar surgió de manera holística con el objetivo de abordar los conflictos escolares proponiendo soluciones que contribuyeran a la mejora del ambiente en los centros educativos. Últimamente hemos observado como la convivencia escolar refleja un nivel de deterioro nunca antes visto en nuestras escuelas e institutos (Merma-Molina et al., 2019; Fernández Guerrero et al., 2021).

Como consecuencia de esta situación, las distintas consejerías educativas de las Comunidades Autónomas han incrementado de manera significativa la puesta en marcha de programas y proyectos educativos encaminados a prevenir y reducir la conflictividad escolar (Viana, 2019), promoviendo estrategias educativas enfocadas a la prevención de conflictos escolares. En este sentido se ha originado un cambio cualitativo importante desde el prisma de la administración educativa y organización de los centros escolares (Andrades-Moya, 2020; Costa et al., 2020; Villanueva-Sierra \& Criado-Avellaneda, 2021). 
Estamos de acuerdo con Toscano et al. (2019), que la calidad docente es un factor estrechamente relacionado con el rendimiento académico, obviamente esta calidad educativa viene promovida por el buen ambiente educativo que en un centro escolar se respira. La armonía en un centro escolar está determinada en parte por las buenas relaciones existentes en un centro escolar entre los miembros de la comunidad educativa. La convivencia escolar es abordada desde diferentes enfoques y no siempre confluyen unanimidad en sus concepciones. Ascorra et al. (2016), Uruñuela (2018) y Fierro-Evans \& Carbajal-Padilla, (2019) hablan de convivencia respetuosa con los derechos humanos y de convivencia en positivo.

La mejora de la convivencia escolar implica compromiso de toda la comunidad educativa para con el alumnado predispuesto a arremeter situaciones de conflictos a través de soluciones autocompositivas (Sánchez et al., 2019) y teniendo en cuenta que las sociedades modernas occidentales exigen estudiantes capacitados para desenvolverse en una sociedad en donde la violencia se incrementa continuamente (Rizo \& Picornell, 2017).

Ahora bien, la convivencia puede verse fracturada por múltiples circunstancias: maltrato psicológico, castigos físicos, violencia verbal, acoso, etc., etc. (Galtung \& Dietrich, 2013). Por otra parte, Pérez-Serrano y Pérez-de-Guzmán, (2011), Brandoni, (2017), Vizcarra et al. (2018), Medina \& Villarreal, (2019), Garaigordobil, (2019), Zepeda, (2020), analizan un variado elenco de tipos de conductas conflictivas escolares que de alguna manera conllevan elevados costes psicológicos en la personalidad de jóvenes que manifiestan baja autoestima (Ormart, 2019). El abuso de poder y la desigualdad entre estudiantes son las bases de esta conflictividad (Garaigordobil \& Oñederra, 2010; Ortega-Ruiz et al., 2016; Torrego, 2018 ).

En este estudio, se concibe la existencia de conflictividad escolar cuando una conducta u omisión de esta realizada intencionadamente, induce daño a otro miembro de la comunidad educativa ya sea estudiante, docente u otro personal (Álvarez-García et al., 2008). Generalmente, las conductas violentas son aprendidas y siempre persiguen imponer, manipular o intimidar (Campos et al., 2015). Las conductas que alteran el ambiente escolar en las aulas son clasificadas por Dobarro et al. (2016) de la siguiente manera: disrupción, violencia verbal, violencia física, exclusión social, violencia a través de las tecnologías de la información y de la comunicación (TIC) y violencia profesor alumnado.

Las conductas indeseadas más habituales que los docentes tienen que soportar y padecer, son los comportamientos disruptivos en el aula, realizada de manera intencionada (Jurado et al., 2020). La disrupción es una conducta tóxica que interrumpe continuamente el proceso de enseñanza- aprendizaje. Hablar a destiempo, levantarse sin permiso del profesor o profesora, molestar constantemente o interrumpir las explicaciones del profesorado, etc., son conductas disruptivas (Álvarez-García et al., 2012).

La violencia física directa se da en los centros educativos con relativa frecuencia, empujones, patadas y peleas entre los estudiantes, generalmente fuera del aula en los pasillos en los cambios de clase y en momentos de receso. La violencia física indirecta, sucede cuando el perjuicio es causado sobre las pertenencias de estudiantes o profesorado o sobre inmuebles y materiales del centro (Campos et al., 2015; Estrada \& Mamani, 2020). Aunque parezca poco probable este tipo de violencia indirecta se ha detectado en estudiantes de enseñanza primaria de 10,11, y 12 años (Zúñiga et al., 2019). 
Las ofensas o poner apodos a estudiantes y profesorado, generar rumores negativos o hablar mal de algún miembro de la comunidad educativa, son modelos de violencia verbal (Domínguez-Alonso et al., 2019). Se puede realizar de manera presencial, es decir, violencia verbal directa y no presencial o violencia verbal indirecta, lógicamente puede ir dirigida hacia los docentes (Nieto et al., 2018).

Otro tipo de violencia que surge en los entornos educativos como consecuencia de la creciente e intensa llegada de población migrante es la exclusión. Trabajos como el de Pachter et al. (2010), Brandoni (2017) y Cabero (2016), analizan los indicadores típicos de esta exclusión social que se focalizan y visibilizan en el espacio escolar. Posteriormente Rizo-Arias (2019) y Escarbajal et al. (2015) exponen analizando los datos obtenidos en su investigación que el nivel de formación de los familias, el lugar de residencia, la nacionalidad, la religión, la etnia, la indumentaria y el aspecto físico son indicadores determinantes de discriminación social y educativa.

Por todo ello, la investigación se ha planteado dos objetivos, el primero, analizar la percepción que tienen los estudiantes de secundaria sobre la violencia verbal, y el segundo, analizar las acciones y la percepción de violencia verbal entre estudiantes y profesorado, y entre los propios estudiantes.

\section{Metodología}

\section{Participantes}

Se ha contado con una muestra incidental de 1069 estudiantes de ESO, pertenecientes a cinco centros de la provincia de Málaga. Tres de estos centros son de zona urbana y el resto de zona rural. La distribución por cursos de muestra en la tabla 1.

Tabla 1.

Porcentaje de Participación por Cursos

\begin{tabular}{rcr}
\hline Cursos & Estudiantes & \% Total \\
\hline $1^{\circ} \mathrm{ESO}$ & 297 & $28 \%$ \\
$2^{\circ} \mathrm{ESO}$ & 267 & $25 \%$ \\
$3^{\circ} \mathrm{ESO}$ & 232 & $22 \%$ \\
$4^{\circ} \mathrm{ESO}$ & 273 & $26 \%$ \\
\hline
\end{tabular}

\section{Diseño}

Se llevó a cabo un estudio típico de la metodología cuantitativa de corte descriptivo, concretamente un estudio tipo encuesta. Se analizó la fiabilidad, validez y utilidad de la prueba. Por otra parte, se realizó un análisis para comprobar las posibles diferencias existentes por cursos y por género de las variables analizadas.

\section{Instrumento y variables}

Para recoger los datos se utilizó la escala CUVE-ESO (Dobarro et al., 2016). Este instrumento es un cuestionario autoaplicado que consta de 44 ítems acompañado de una 
escala tipo Likert con valores comprendidos entre 1 y 5 , siendo 1 "Nunca", 2 "Pocas Veces", 3, "Algunas Veces", 4 "Muchas Veces" y 5 "Siempre.

El estudio se centra en el análisis de las escalas descritas en la tabla 2.

Tabla 2.

Descripción de las Escalas Generales

\begin{tabular}{ll}
\hline Descripción & Escala \\
\hline Violencia Verbal entre Estudiantes & VVAA \\
Violencia Verbal de alumnado a profesorado & VVAP \\
\hline Álvarez-García et al. (2017) &
\end{tabular}

En la tabla 3 se describe el contenido de las distintas subescalas que componen el CUVEESO.

Tabla 3.

Descripción de las Subescalas

\begin{tabular}{ll}
\hline Subescalas & \multicolumn{1}{c}{ Descripción } \\
\hline vvaa1 & El alumnado pone motes molestos a sus compañeros/as \\
vvaa2 & Hay estudiantes que extienden rumores negativos acerca de compañeros/as \\
vvaa3 & Los estudiantes hablan mal unos de otro \\
vvaa4 & El alumnado insulta a sus compañeros o compañeras \\
vvap1 & El alumnado habla con malos modales al profesorado \\
vvap2 & El alumnado falta al respeto al profesorado en el aula \\
vvap3 & Los estudiantes insultan a profesores o profesoras \\
\hline Álvarez-García et al., (2017)
\end{tabular}

\section{Procedimiento}

El procedimiento seguido en la investigación sigue las fases clásicas del mismo. Se inició con la solicitud de autorización a las distintas direcciones de los centros para la administración del instrumento. Una vez conseguida se procedió a contactar con tutores, tutoras y profesorado, para informarles cuál era el objetivo de la investigación. Se instruyó a los docentes encargados de aplicar el instrumento a los distintos cursos en cada centro.

Se explicó el modo de responder el cuestionario a los estudiantes, advirtiéndoles que para contestar al cuestionario tendrían alrededor de 25 minutos. Para aquellos estudiantes que pudieran presentar algún tipo de dificultad de comprensión lectora podían tener ayuda por parte del docente supervisor.

\section{Análisis y resultados}

Se llevó a cabo un análisis descriptivo de las variables de interés. Las propiedades psicométricas para verificar la consistencia interna fueron calculadas a partir del coeficiente alfa de Cronbach y coeficiente omega de McDonald. Igualmente, se confirmó la estructura 
latente de la medición aplicando un análisis factorial confirmatorio según la estructura de ocho factores que indican (Dobarro et al., 2016) para valorar el ajuste del modelo se utilizaron los índices Chicuadrado con relación a sus grados de libertad, RMSEA, SRMR, TLI y CFI (Kaplan, 2009; Hormigo, 2014; Shi et al., 2018). Seguidamente se calcularon las puntuaciones de cada participante para las siete subescalas promediando sus puntuaciones en los ítems correspondientes a dichos factores. A partir de estos resultados se analizó el perfil de respuesta de los participantes de forma descriptiva para analizar la aparición de la frecuencia de estas conductas, siguiendo los objetivos de estudio. Por último, se exploró la posible existencia de diferencias en función del género y del curso de los participantes. Los análisis se realizaron con la librería Jamovi de R (The Jamovi Project 2021).

Los coeficientes de consistencia interna para la escala global fueron de .81 para el alpha de Cronbach y de .81 para el Omega de McDonald. Los valores de fiabilidad para las siete subescalas son aceptables con un mínimo en alfa de 0.77 y de 0.78 para omega y máximos de 0.81 para alfa y 0.82 para omega.

Con respecto a la escala VVAA observamos que el promedio de la frecuencia percibida es elevado $(M=2.68 ; d t=0.90)$ presentando un sesgo positivo (sesgo=0.32). En esta escala, la subescala más alta la presenta vvaz los estudiantes hablan mal unos de otros. La escala VVAP disminuyó ligeramente el resultado $(M=2.23 ; \mathrm{dt}=0.87)$ presentando un sesgo positivo (sesgo=0.65) y se concluye que la mayoría de los estudiantes tienen un nivel de percepción elevado de la violencia verbal tanto entre los mismos estudiantes y de estudiantes hacia el profesorado. Los porcentajes de la escala VVAA aparecen en la figura 1 mientras que los porcentajes de la escala VVAP aparecen en la figura 2.

Figura 1.

Porcentajes del Factor VVAA

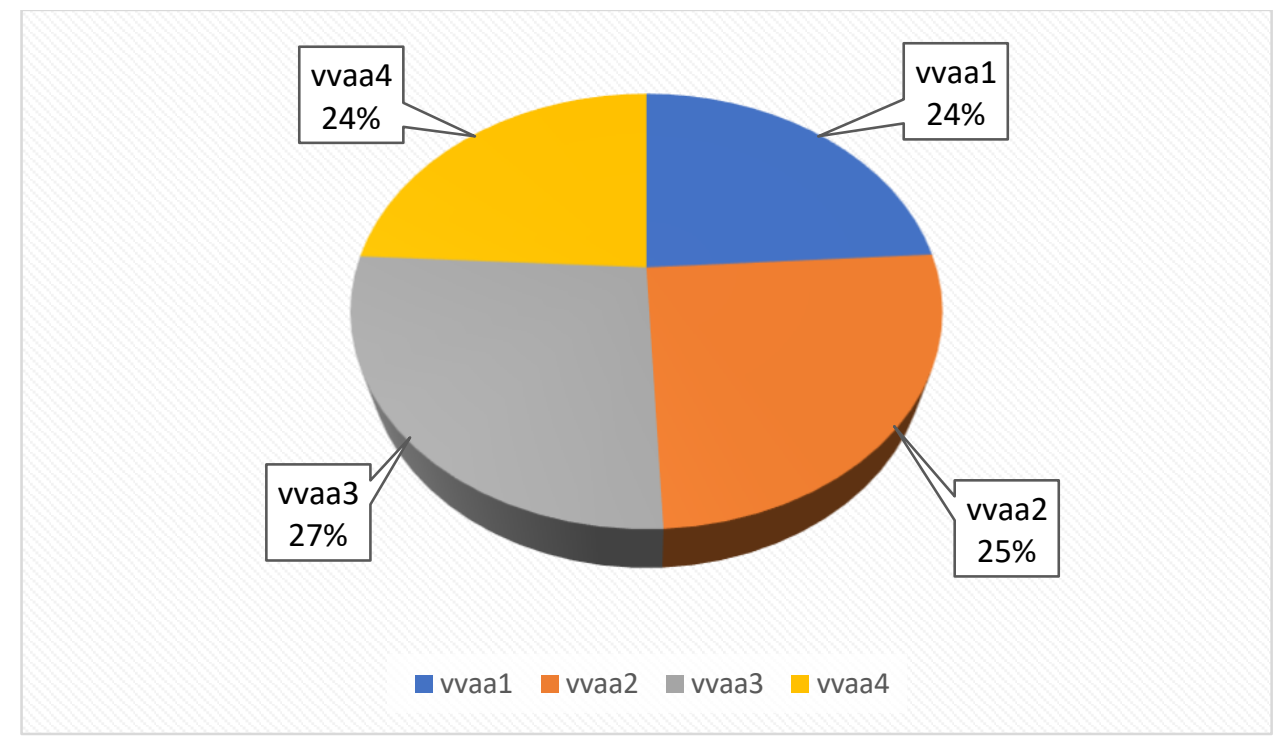


La relación existente entre todas las subescalas es positiva (0.52). Puede observarse la escasa diferencia porcentual entre las subescalas en dónde destaca vvaa3 (27\%) como ya hemos afirmado anteriormente. En lo que respecta a la escala VVAP existe una escasa diferencia porcentual entre vvap1 (37\%) el alumnado habla con malos modales al profesorado vap2 (36\%) el alumnado falta al respeto al profesorado. La conducta menos percibida por los estudiantes es vvap3 (27\%) los estudiantes insultan a profesores y profesoras.

Figura 2.

Porcentajes del Factor VVAP

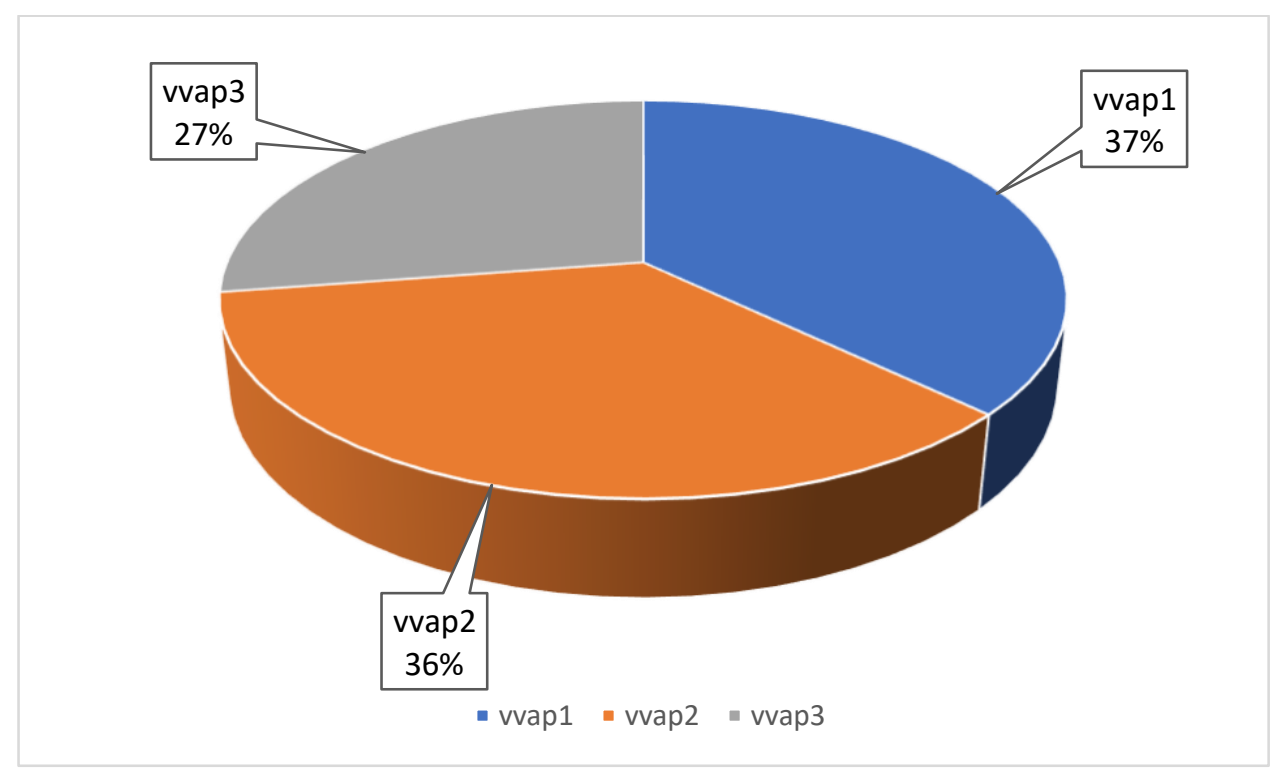

En la tabla 4 se incluye la matriz de correlación de las variables asignadas a las subescalas. Las variables con un valor más elevado de correlación son la vvaaz, los estudiantes hablan mal unos de otros con vvaa2, hay estudiantes que extienden rumores negativos acerca de compañeros y compañeras; y vva4, el alumnado insulta a sus compañeros o compañeras con vva3 los estudiantes hablan mal unos de otros. Mientras que con respecto a los docentes las más correlacionadas son vvap2 (0.31) el alumnado falta al respeto al profesorado en el aula con vvap1 (0.33), el alumnado habla con malos modales al profesorado. El resto de los valores están por debajo de 0.50 , pero todas con valores positivos lo que quiere decir que si aumenta una subescala aumentará la otra. 
Tabla 4

Matriz de correlación de subescalas

\begin{tabular}{lrrrrrrr}
\hline & vvaa1 & vvaa2 & vvaa3 & vvaa4 & vvap1 & vvap2 & vvap3 \\
\hline vvaa1 & 1 & & & & & & \\
vvaa2 & 0.31 & 1 & & & & & \\
vvaa3 & 0.32 & 0.57 & 1 & & & & \\
vvaa4 & 0.32 & 0.41 & 0.56 & 1 & & & \\
vvap1 & 0.26 & 0.31 & 0.38 & 0.36 & 1 & & \\
vvap2 & 0.22 & 0.30 & 0.39 & 0.40 & 0.66 & 1 & \\
vvap3 & 0.26 & 0.29 & 0.35 & 0.39 & 0.47 & 0.48 & 1 \\
\hline
\end{tabular}

En cuanto a la estructura factorial del instrumento, se han obtenido siete factores y un valor de Chi-cuadrado de 420.48 ( $g l .=14 ; p=.001$ ), lo que significa una razón de chi-cuadrado/grados de libertad de 30.30. Así pues, puede considerarse como un ajuste aceptable. Lo mismo ocurre con los índices de ajuste RMSEA=0.16 y $\mathrm{SRMR}=0.07$, que indican un ajuste adecuado.

Todas las subescalas vvaa1, vvaa2, vvap1, vvap2 están con una puntuación poco significativa, salvo la subescala vvap3. La frecuencia de la subescala vvaa3, los estudiantes hablan mal unos de otros, (media $=2.85, \mathrm{dt}=1.25$ ). Los estudiantes insultan a profesores y profesoras que es la que presenta menor percepción (media=1.84; dt=0.96). Todas las subescalas presentan sesgo positivo.

Otro aspecto a considerar es la existencia de posibles diferencias entre género. Puesto que en la distribución de las dimensiones no pueden considerarse normales aplicamos la prueba Kruskall-Wallis. Los resultados no mostraron diferencias significativas en función del género de los participantes a un nivel de confianza del $99 \%$ en todas las subescalas salvo en vvaa1 y vvaa4.

Tabla 5.

Kruskal-Wallis por Género (VVAA y VVAP)

\begin{tabular}{lrrr}
\hline & $\boldsymbol{X}^{2}$ & df & p \\
\hline vvaa1 & 3.99 & 1 & 0.046 \\
vvaa2 & 19.36 & 1 & $<.001$ \\
vvaa3 & 30.36 & 1 & $<.001$ \\
vvaa4 & 7.07 & 1 & 0.008 \\
vvap1 & 21.68 & 1 & $<.001$ \\
vvap2 & 15.71 & 1 & $<.001$ \\
vvap3 & 28.11 & 1 & $<.001$ \\
\hline
\end{tabular}

En la siguiente fase del proceso de análisis indagamos en la existencia de posibles diferencias entre cursos. En este caso, se registran diferencias significativas tal como puede comprobarse en la prueba de Kruskal-Wallis de la tabla 6. En vvap2, se obtuvo, diferencia significativa a un nivel de significación de 0.05 de las subescalas la dimensión de la dimensión violencia verbal. 
Tabla 6.

Kruskal-Wallis por Cursos (VVAA y VVAP)

\begin{tabular}{lrrr}
\hline & $\chi^{2}$ & df & p \\
\hline vvaa1 & 12.62 & 3 & 0.006 \\
vvaa2 & 8.80 & 3 & 0.032 \\
vvaa3 & 22.42 & 3 & $<.001$ \\
vvaa4 & 10.27 & 3 & 0.016 \\
vvap1 & 61.33 & 3 & $<.001$ \\
vvap2 & 75.45 & 3 & $<.001$ \\
vvap3 & 40.60 & 3 & $<.001$ \\
\hline
\end{tabular}

Para indagar las diferencias en concreto, se calcularon los estadísticos descriptivos del promedio, desviación típica por curso y subescalas para la dimensión VVAA y para la dimensión VVAP. Los resultados de las subescalas presentan un patrón común en forma de arco invertido que consiste en un valor medio más bajo en primer curso, para experimentar un incremento en segundo y tercero y volver a bajar en cuarto curso. Las diferencias son estadísticamente significativas, aunque los tamaños de efecto son relativamente bajos.

Tabla 7.

Descriptivos por Cursos (VVAA)

\begin{tabular}{rlrrrr}
\hline & IdCurso & vvaa1 & vvaa2 & vvaa3 & vvaa4 \\
\hline $\mathrm{N}$ & $1^{\circ} \mathrm{ESO}$ & 303 & 303 & 303 & 303 \\
& $2^{\circ} \mathrm{ESO}$ & 267 & 267 & 267 & 267 \\
& $3^{\circ} \mathrm{ESO}$ & 232 & 232 & 232 & 232 \\
\multirow{4}{*}{ Media } & $4^{\circ} \mathrm{ESO}$ & 267 & 267 & 267 & 267 \\
& $1^{\circ} \mathrm{ESO}$ & 2.51 & 2.74 & 2.77 & 2.66 \\
& $2^{\circ} \mathrm{ESO}$ & 2.57 & 2.73 & 2.81 & 2.64 \\
& $3^{\circ} \mathrm{ESO}$ & 2.80 & 2.85 & 3.21 & 2.69 \\
& $4^{\circ} \mathrm{ESO}$ & 2.46 & 2.52 & 2.69 & 2.37 \\
$\mathrm{DT}$ & $1^{\circ} \mathrm{ESO}$ & 1.19 & 1.13 & 1.21 & 1.27 \\
& $2^{\circ} \mathrm{ESO}$ & 1.08 & 1.17 & 1.19 & 1.20 \\
& $3^{\circ} \mathrm{ESO}$ & 1.18 & 1.32 & 1.37 & 1.26 \\
& $4^{\circ} \mathrm{ESO}$ & 1.04 & 1.08 & 1.18 & 1.08 \\
\hline
\end{tabular}

Nota. Fuente elaboración propia.

Tabla 8.

Descriptivos por Cursos (VVAP)

\begin{tabular}{llrrr}
\hline & IdCurso & vvap1 & vvap2 & vvap3 \\
\hline $\mathrm{N}$ & $1^{\circ} \mathrm{ESO}$ & 303 & 303 & 303 \\
& $2^{\circ} \mathrm{ESO}$ & 267 & 267 & 267 \\
& $3^{\circ} \mathrm{ESO}$ & 232 & 232 & 232 \\
& $4^{\circ} \mathrm{ESO}$ & 267 & 267 & 267 \\
Media & $1^{\circ} \mathrm{ESO}$ & 2.11 & 1.95 & 1.59
\end{tabular}




\begin{tabular}{rlrrr}
\hline & IdCurso & vvap1 & vvap2 & vvap3 \\
\hline \multirow{4}{*}{ DT } & $2^{\circ} \mathrm{ESO}$ & 2.65 & 2.51 & 1.92 \\
& $3^{\circ} \mathrm{ESO}$ & 2.71 & 2.70 & 1.95 \\
& $4^{\circ} \mathrm{ESO}$ & 2.57 & 2.43 & 1.93 \\
& $1^{\circ} \mathrm{ESO}$ & 1.00 & 1.04 & 0.92 \\
& $2^{\circ} \mathrm{ESO}$ & 1.02 & 1.12 & 0.98 \\
& $3^{\circ} \mathrm{ESO}$ & 1.14 & 1.17 & 0.98 \\
& $4^{\circ} \mathrm{ESO}$ & 0.91 & 0.96 & 0.94 \\
\hline
\end{tabular}

Destaca la escala VVAA en $3^{\circ}$ de ESO, enfocada a la violencia verbal entre alumnado, subescala vvaa3, $(M=3.21)$ los estudiantes hablan mal unos de otros, es con diferencia la subescala más percibida en los cursos de ESO, sin dejar a un lado la subescala vvaa1, el alumnado pone motes a sus compañeros o compañeras $(M=2.80)$; la subescala vvaaz $(M=2.85)$, hay estudiantes que extienden rumores negativos acerca de compañeros y compañeras y la subescala vvaa4, el alumnado insulta a sus compañeros y compañeras $(M=2.69)$, todas ellas próximas o por encima del valor promedio. La puntuación mínima es 1 y la máxima es de 5. Lo mismo sucede con la dimensión VVAP, la subescala vvap1, el alumnado habla con malos modales al profesorado $(M=2.71)$; la subescala vvap2, el alumnado falta al respeto al profesorado en el aula $(M=2.70)$ pero disminuye en la subescala vvap3, los estudiantes insultan a los profesores o profesoras $(M=1.95)$.

\section{Discusión y conclusiones}

Hay que subrayar que el tamaño de la muestra y su representatividad minimiza las posibilidades de generalización y alcance, aunque la escala de valores psicométricos es aceptable. La consistencia interna calculada con el alfa de Cronbach es considerada como muy admisible. Además, el análisis factorial confirmatorio apoya la idea de un modelo latente de ocho dimensiones que explicaría una parte destacable de la variabilidad de los datos. El aumento de registros sería la solución para proceder a una generalización más completa. Se tenía la pretensión de ampliarla, pero la pandemia mundial por el COVID19 lo impidió.

Los centros de ESO son un foco donde se genera la violencia que muy posiblemente tiene su origen en entornos sociales y familiares (Pazo, 2020). La violencia generada en contextos sociales, familiares y escolares redunda negativamente en el rendimiento académico del alumnado, siendo en ocasiones causa relevante para el abandono precoz del sistema educativo y del consiguiente fracaso escolar (Álvarez-Gómez, 2019; Zepeda, 2020). En la mayoría de los casos estas conductas están normalizadas entre alumnado y hacia profesorado, suelen ser habituales y cotidianas en los centros educativos. Este tipo de comportamiento se realiza fuera del aula e incluso en redes sociales, lo cual complejiza las posibles medidas de intervención en el espacio educativo.

La violencia verbal en nuestro centros son una constante y los resultados obtenidos en nuestro estudio son comparables a otras investigaciones (Dobarro et al., 2016; Nieto et al., 2018; Conde \& Delgado-García, 2020;Fernández Guerrero et al., 2021. El alumnado es 
consciente de que hay eventos e incidencias recurrentes en los que manifiestan violencia verbal explícita entre ellos, principalmente hablando mal los unos de los otros y con repercusión en el clima escolar. En nuestro estudio obtuvimos diferencia significativa a un nivel de significación de 0.05 de las subescalas la dimensión de violencia verbal. Por lo que con estos resultados consideramos que sí existen evidencias suficientes como para considerar que existen diferencias entre alumnos y alumnas. A la luz de los resultados se concluye que la mayoría de los estudiantes de ESO tienen un nivel de percepción alto de la violencia verbal tanto entre estudiantes como de estudiantes hacia el profesorado, llegando incluso a contemplarse como una conducta normalizada o, en su defecto, una naturalización banalizada de determinadas pautas interactivas y de comunicación. En este sentido, no podemos obviar que la violencia verbal se manifiesta en las dimensiones y subdimensiones exploradas (poner motes, estigmatizar, extender rumores, hablar mal de los compañeros, faltas de respeto al profesorado, etc.). Principalmente se observa que el alumnado presenta determinados modales incívicos hacia el profesorado junto con falta de respeto e insultos. Esto se vincula con la expansión de una forma bronca de expresarse que ya de por sí implica falta de respeto y tienen consecuencias significativas en la configuración del clima escolar. Igualmente, existe una alta percepción de este conflicto verbal tanto entre estudiantes como de estudiantes hacia el profesorado y subrayamos que sí existen evidencias suficientes como para constatar que existen diferencias de género entre alumnos y alumnas en esas subescalas.

Se ha identificado que las conductas violentas pueden desembocar en acoso escolar, situación problemática a veces de muy difícil detección y visibilización explícita si se capilariza a través de distintos medios y dispositivos (Ortega-Ruíz \& Córdoba-Alcaide, 2017). Al igual que cualquier otro tipo de violencia, los estudiantes expuestos a violencia ven reducida su capacidad integrativa en su ambiente como así lo argumentan en su estudio Sandoval et al, (2017). Por su parte, en otros estudios realizados (Domínguez-Alonso \& Portela, 2020) se ha constatado una mayor incidencia en el género masculino que en el femenino en lo relativo a la violencia verbal hacia estudiantes y profesorado. Nuestros resultados no convergen con los hallados por Domínguez-Alonso y Portela (2020), ya que se observa diferencia alguna entre hombres y mujeres y diferencias por edades. Sí podemos afirmar que los cursos intermedios de ESO, $2^{\circ}$ y $3^{\circ}$, presentan una mayor violencia verbal.

Como fórmula proactiva de resolución de conflictos entre iguales hemos propuesto la mediación escolar. Ahora bien, la podemos concebir como una estrategia didáctica especializada necesaria y adecuada para con los estudiantes, no siendo útil para cuando la violencia verbal va dirigida hacia los docentes. En todo caso, su inclusión requiere de la interdependencia y confluencia positiva de distintas medidas y acciones donde resulta clave las aportaciones del profesional de la orientación educativa y su compromiso ineludible con la mejora y la formación permanente en convivencia escolar.

Con los resultados obtenidos en el estudio se logra alcanzar el objetivo general inicial de analizar cuantitativamente la percepción que tienen los estudiantes de la violencia verbal existente en ESO y hemos cuantificado la existente entre estudiantes y hacia el profesorado para proponer la mediación escolar como estrategia proactiva y positiva en la gestión y resolución de conflictos. 
La mediación lleva intrínseca los principios de voluntariedad, igualdad, confidencialidad, presupone una acción proactiva que genera corresponsabilidad y participación. Fomenta la empatía entre estudiantes, el compromiso y la puesta en práctica de toda una serie de parámetros necesarios para la mejora del clima escolar (Bueno, 2018; Rotila, 2019). No podemos dejar de enfatizar la necesidad de la mediación entre iguales como aquella herramienta pedagógica que promueve un ambiente constructivo y estimulante en la emergencia y desarrollo de valores sociales, comunicación, tolerancia y autocontrol positivos (Pérez-Serrano \& Pérez-de-Guzmán, 2011), siendo un motor de cambio e innovación que vehicule de forma proactiva y empática el aprendizaje del ser y del convivir de los más jóvenes en los centros educativos (Bernal, 2012; Torrego, 2018; García-Raga et al., 2019).

En definitiva, debemos seguir cultivando la mediación escolar entre estudiantes como una estrategia proactiva de gestión positiva de conflictos por ser un procedimiento autocompositivo y que se imbrica adecuadamente con una mentalidad de crecimiento y conciencia de generar identidades pacíficas y responsables (Boqué, 2018; Moreta, 2018). Comprobar la eficacia de esta medida supone la apertura de otra línea de investigación en un centro que haya implementado la mediación escolar entre iguales y constatar posteriormente su efectividad. Finalmente, resulta esencial reforzar las acciones de orientación educativa en materia de promoción de la mediación, así como la optimización de los medios y recursos didácticos para que el profesorado pueda formarse, actualizar y revitalizar sus prácticas docentes.

\section{Referencias}

Álvarez-García, D., Álvarez, L., Nuñez, J. C., González-Pienda, J. A., González-Castro, P., \& Rodríguez, C. (2008). Estudio del nivel de violencia escolar en siete centros asturianos de Educación Secundaria. Aula Abierta, 36(1), 89-96. https://dialnet.unirioja.es/descarga/articulo/2856322.pdf

Álvarez-García, D., Núñez, J. C., \& Dobarro, A. (2012). Manual de Cuvez. ALBOR-COHS. https://studylib.es/doc/6031188/manual-de-referencia--versión-2.2---grupo-albor-cohs

Álvarez-García, D., Núñez, J. C., \& Dobarro, A. (2017, December 14). CUVE3ESO: Manual de referencia [Manual]. https://studylib.es/doc/6031188/manual-de-referencia--versión-2.2---grupo-albor-cohs

Álvarez-Gómez, B. P. (2019). Violencia escolar y el desarrollo socio afectivo de los niños y niñas de la unidad educativa unidad popular periodo 2019 [Proyecto de investigación]. BABAHOYO: UTB, 2019. http://dspace.utb.edu.ec/handle/49000/7146

Andrades-Moya, J. (2020). Convivencia escolar en Latinoamérica: Una revisión bibliográfica. Revista Electrónica Educare, 24 (2)(2), 19-32. https://doi.org/10.15359/ree.24-2.17

Bernal, T. (2012). La mediación en escena. EOS.

Boqué, M. C. (2018). La mediación va a la escuela. Hacia un buen plan de convivencia en el centro. Narcea.

Brandoni, F. (2017). Conflictos en la escuela. EDUNTREF. 
http://eduntref.com.ar/magento/pdf/conflictos-en-la-escuela-digital.pdf

Bueno, A. (2018, June 15). Mediación y menores: mediación escolar [TFM]. Universidad de Valladolid. Facultad de Ciencias Sociales, Jurídicas y de la Comunicación. http://uvadoc.uva.es/handle/10324/30759

Cabero, J. (2016). La educación a distancia como estrategia de inclusión social y educativa. Universidad Nacional Autónoma de México. https://idus.us.es/xmlui/handle/11441/34247

Campos, J. A., Tass, K. O. M. El, \& Cruz, G. de C. (2015). Violência escolar: relações entre bullying e a educação física. Revista Espacios, 36(11), E-1. https://www.revistaespacios.com/a15v36n11/153611E1.html

Carmona, M. . A., Castillón, L., \& Gutiérrez-Gómez, R. (2020). Los conflictos escolares como factor de riesgo en el rendimiento académico y la deserción escolar. Revista RedCA, 3(7), 82-100. https://convergencia.uaemex.mx/index.php/revistaredca/article/view/14703

Conde, S., \& Delgado-García, M. (2020). Percepciones del alumnado sobre diferentes tipos de violencia. Adaptación y validación del CUVE3 -ESO al contexto Universitario. Revista de Investigación Educativa, 38(2), 567-581. https://doi.org/DOI: http://dx.doi.org/10.6018/rie.364431

Costa, V., Teyes, R., \& Zamora., M. (2020). Estudio comparativo entre los programas de prevención de la violencia escolar. In Haciendo ciencia, construimos futuro (pp. 580592). Universidad de Zulia. https://scholar.google.es/scholar?hl=es\&as_sdt=0\%2C $5 \& q=H a c i e n d o+c i e n c i a+c o n s t r u i$ mos+futuro+Teyes\&btnG=

Dobarro, A., Álvarez García, D., \& Núñez, J. C. (2016). CUVE3:Instrumentos para evaluar la violencia escolar. International Journal of Developmental and Educational Psychology. Revista INFAD de Psicología., 5(1), 487. https://doi.org/10.17060/ijodaep.2014.n1.v5.710

Domínguez-Alonso, J., López-Castedo, A., \& Nieto-Campos, B. (2019). Violencia escolar: diferencias de género en estudiantes de secundaria. Revista Complutense de Educación, 30(4), 1031-1044. https://doi.org/https://doi.org/10.5209/rced.59997

Domínguez-Alonso, J. \& Portela, I. (2020). Violencia a través de las TIC: comportamientos diferenciados por género. RIED. Revista Iberoamericana de Educación a Distancia, 23(2), 273. https://doi.org/10.5944/ried.23.2.25916

Escarbajal, A., Sánchez, M., \& Guirao, I. (2015). Factores determinantes del rendimiento académico de alumnos hijos de inmigrantes y autóctonos de origen extranjero en contextos de exclusión social. Revista Sobre La Infancia y La Adolescencia, 9, 31-46. https://doi.org/https://doi.org/10.4995/reinad.2015.3772

Estrada, E. G., \& Mamani, H. J. (2020). Violencia escolar y niveles de logro de aprendizaje en una institución educativa pública de Puerto Maldonado. PURIQ, 2(3), 246-260. https://doi.org/10.37073/puriq.2.3.86

Fernández, M., Suárez, M., Rojo, J., \& Feu, S. (2021). Acoso escolar en Educación Primaria y en Educación Secundaria en Badajoz. Análisis de los factores de riesgos. Revista 
Electrónica Interuniversitaria de Formación Del Profesorado, 24(2). https://doi.org/10.6018/reifop.434581

Fierro-Evans, C., \& Carbajal-Padilla, P. (2019). Convivencia Escolar: Una revisión del concepto. Psicoperspectivas, 18(1), 9-27. https://doi.org/10.5027/psicoperspectivas-Vol18-Issue1fulltext-1486

Galtung, J., \& Dietrich, F. (2013). Pioneer of Peace Research. Springer.

Garaigordobil, M. y Oñederra, J. A. (2010). La violencia entre iguales. Revisión teórica y estrategias de intervención. Piramide.

Garaigordobil, M. (2019). Prevention of cyberbullying: personal and family predictive variables of cyber-aggression Prevención del cyberbullying: variables personales y familiares predictoras de ciberagresión. Revista de Psicología Clínica Con Niños y Adolescentes, 6(3), 2019-2028. https://doi.org/10.21134/rpcna.2019.06.2.1

García-Raga, L., Boqué, M. C., \& Grau, R. (2019). Valoración de la mediación escolar a partir de la opinión de alumnado de educación secundaria de Castellón, Valencia y Alicante (España). Profesorado, Revista de Currículum y Formación Del Profesorado, 23(1), 103-119. https://doi.org/10.30827/profesorado.v23i1.9146

Hormigo, A. L. (2014). Introducción a las ecuaciones estructurales en AMOS y R. https://masteres.ugr.es/moea/pages/curso201314/tfm1314/tfm-

septiembre1314/memoriamasterantonio_lara_hormigo/!

Iriarte, C., Ibarrola-García, S., \& Aznárez-Sanado, M. (2020). Propuesta de un instrumento de evaluación de la mediación escolar (CEM). Revista Española de Pedagogía, 276(78), 300326. https: // www. jstor .org / stable / 26915244

Jurado, P., Lafuente, A., \& Justiniano, M. D. (2020). Conductas disruptivas en Educación Secundaria Obligatoria: análisis de factores intervinientes. Contextos Educativos. Revista de Educación, 25(25), 219-236. https://doi.org/10.18172/con.3827

Kaplan, D. (2009). Structural equation modeling: foundations and extensions. Segunda edición. Thousand Oaks, CA: Sage. https://doi.org/doi:10.4135/9781452226576.

Medina, M. A. G., \& Villarreal, D. C. T. (2019). Violencia escolar en bachillerato: algunas estrategias para su prevención desde diferentes perspectiva Educación. Teoría de La Educación. Revista Interuniversitaria USAL., 123-147. https://doi.org/DOI: http://dx.doi.org/10.14201/teri.19616

Moreta, M. (2018). Educar en el conflicto: Mediación escolar y gestión de conflictos en educación secundaria.

https://repositorio.unican.es/xmlui/bitstream/handle/10902/14432/MoretaAbascalMart a.pdf? sequence $=1$ \&isAllowed $=y$

Nieto, B., Portela, I., López, E., \& Domínguez, V. (2018). Violencia verbal en el alumnado de Educación Secundaria Obligatoria. The British Journal of Psychiatry, 1(1), 5-14. https://doi.org/doi:10.30552/ejihpe.v8i1.221 Violencia 
Ormart, E. B. (2019). Violencia escolar y planificación educativa. Nodos y Nudos: Revista de La Red de Calificación de Educadores, 6(46), 25-36. https://dialnet.unirioja.es/servlet/articulo?codigo=7143984\&orden=0\&info=link

Ortega-Ruíz, R., \& Córdoba-Alcaide, F. (2017). El modelo construir la convivenci para prevenir el acoso y el ciberacoso escolar. Innovación Educativa, 27, 19-32. https://doi.org/https://doi.org/10.15304/ie.27.4287

Ortega-Ruiz, R., Rey, R. Del, \& Casas, J. A. (2016). Revista iberoamericana de evaluación educativa. In Revista Iberoamericana de Evaluación Educativa (Vol. 6, Issue 2). https://revistas.uam.es/index.php/riee/article/view/3406

Pachter, L. M., Bernstein, B. A., Szalacha, L. A., \& Coll, C. G. (2010). Perceived racism and discrimination in children and youths: An exploratory study. Health \& Social Work, 35(1), 61-70. https://doi.org/https://doi.org/10.1093/hsw/35.1.61

Pazo, J.(2020). Entre la violencia familiar y la violencia escolar. Investigaciones Sociales, 22(42), 19-36. https://doi.org/10.15381/is.v22i42.17477

Pérez-Serrano, G., \& Pérez-de-Guzmán, M. V. (2011). Aprender a convivir: El conflicto como oportunidad de crecimiento. Narcea.

Rizo-Arias, L. J. (2019). Índices de exclusión educativa en el etapa de la ESO en la provincia de Salamanca. Papeles Salmantinos de Educación, 23, 9-29. https://revistas.upsa.es/index.php/papeleseducacion/article/view/21

Rizo, L. J., \& Picornell, A. (2017). Percepciones del Profesorado respecto al bullyingy su relación con la desafección y el fracaso escolar en la Provincia de Salamanca. Prisma Social, 17, 396-414. https://revistaprismasocial.es/article/view/1290/1356

Sánchez, A., Alexander, R., López, A., \& Patricia, A. (2019). La educación en mediación escolar como escenario de formación ciudadana Education in school mediation as a scenario for $\begin{array}{llll}\text { citizen education } & \text { Contenido: } & \text { Vol. } & 40\end{array}$ https://www.revistaespacios.com/a19v40n21/a19v40n21p01.pdf

Sandoval, J., Abril, A., \& Leal, H. (2017, March). Violencia en la escuela: creencias y percepciones de docentes y estudiantes [Comunicación]. Actas del Congreso Nacional de investigación Educativa. http://www.comie.org.mx/congreso/memoriaelectronica/v14/doc/1895.pdf

Shi, D., Maydeu-Olivares, A., \& DiStefano, C. (2018). The Relationship Between the Standardized Root Mean Square Residual and Model Misspecification in Factor Analysis Models. Multivariate Behavioral Research, 53(5), 676-694. https://doi.org/10.1080/00273171.2018.1476221

The jamovi project (2021). jamovi (Version 1.6) [Computer Software]. Retrieved from https://www.jamovi.org.

Torrego, J. C. (2019, May 23). Desde la mediación de conflictos en centros escolares hacia el modelo integrado de mejora de la convivencia. http://diversidad.murciaeduca.es/orientamur/gestion/documentos/articulo_revisado_c eapa_torrego.pdf 
Torrego, J. C. (Coord). (2018). Mediación de conflictos en instituciones educativas. Manual para la formación de mediadores (8th ed.). Narcea.

Toscano, D. F., Peña-Nivicela, G. E., \& Lucas-Aguilar, G. A. (2019). CONVIVENCIA Y RENDIMIENTO ESCOLAR. In Revista Metropolitana de Ciencias Aplicadas (Vol. 2, Issue 2). https://orcid.org/0000-0001-5104-3615

Uruñuela, P. M. (2018). Trabajar la convivencia en los centros educativos. Una mirada al bosque de la convivencia. . Narcea.

Vázquez-Gutiérrez, R. L. (2019). Objetivos de la Educación para la paz. In Estudios interdiciplinarios: Paz y comunicación. (pp. 378-398). UNESP. https://ng.cl/87h4

Viana, M. I. (2019). 25 años de Mediación Escolar en España: 1994-2019. Cuestiones Pedagógicas, o(27), 11-22. https://doi.org/10.12795/CP.2018.i27.01

Villanueva-Sierra, M. A., \& Criado-Avellaneda, J. (2021). La convivencia positiva en las aulas del segundo ciclo de educación inicial. Pontificia Universidad Católica Del Perú. http://repositorio.pucp.edu.pe/index/handle/123456789/175438

Vizcarra, M. . T., Rekalde, I., \& Macazaga, A. M. . (2018). La percepción del conflicto escolar en tres comunidades de aprendizaje. Magis. Revista Internacional de Investigación En Educación, 10(21), 95-108. https://dialnet.unirioja.es/servlet/articulo?codigo=6516365

Zepeda, M. de los Á. C. (2020). Los conflictos escolares como factor de riesgo en el rendimiento académico y la deserción escolar. Revista RedCA, v. 3, n. 7, pág. 82-100. https://revistaredca.uaemex.mx/article/view/14703

Zúñiga, L. F. S., Rivas, P. L., \& Trevizo, J. G. R. (2019). Percepción de la violencia escolar en el último ciclo de educación primaria. Revista Electrónica Cientifica de Investigación Educativa, Vol. 4 Núm, 1349-1350. https://doi.org/DOI: https://doi.org/10.33010/recie.v4i2.369 